\title{
SARS-CoV-2 receptor binding domain-specific antibodies activate platelets with features resembling the pathogenic antibodies in heparin- induced thrombocytopenia
}

\author{
Wen Zhu \\ Blood Research Center \\ Yongwei Zheng \\ Blood Research Center \\ Mei Yu \\ Blood Research Center \\ Jianhui Wei
}

Fujian Normal University

Yongguang Zhang

Fujian Normal University

Paytsar Topchyan

Blood Research Center

Christine Nguyen

Blood Research Center

Rae Janecke

Blood Research Center

Lisa Baumann Kreuziger

Blood Research Center

Gilbert C. White

Blood Research Center

Parameswaran Hari

Medical College of Wisconsin

Richard Aster

Blood Research Center

Weiguo Cui

Blood Research Center

Shawn Jobe

Blood Research Center

Mary Beth Graham

Blood Research Center 


\section{Demin Wang}

Blood Research Center

Renren Wen ( $\square$ renren.wen@bcw.edu)

Blood Research Center

\section{Research Article}

Keywords: heaprin induced thrombocytopenia, covid-19, platelet-activating antibodies

Posted Date: April 26th, 2021

DOI: https://doi.org/10.21203/rs.3.rs-462080/v1

License: (c) (i) This work is licensed under a Creative Commons Attribution 4.0 International License. Read Full License 


\section{Abstract}

Severe COVID-19 is associated with unprecedented thromboembolic complications. We found that hospitalized COVID-19 patients develop immunoglobulin Gs (IgGs) that recognize a complex consisting of platelet factor 4 and heparin similar to those developed in heparin-induced thrombocytopenia and thrombosis (HIT), however, independent of heparin exposure. These antibodies activate platelets in the presence of TLR9 stimuli, stimuli that are prominent in COVID-19. Strikingly, 4 out of 42 antibodies cloned from IgG1 ${ }^{+}$RBD-binding B cells could activate platelets. These antibodies possessed, in the heavy-chain complementarity-determining region 3 , an $\mathrm{RKH}$ or $\mathrm{Y}_{5}$ motif that we recently described among plateletactivating antibodies cloned from HIT patients. RKH and $Y_{5}$ motifs were prevalent among published RBDspecific antibodies, and 3 out of 6 such antibodies tested could activate platelets. Features of platelet activation by these antibodies resemble those by pathogenic HIT antibodies. B cells with an RKH or $\mathrm{Y}_{5}$ motif were robustly expanded in COVID-19 patients. Our study demonstrates that SARS-CoV-2 infection drives the development of a subset of RBD-specific antibodies that can activate platelets and have activation properties and structural features similar to those of the pathogenic HIT antibodies.

\section{Introduction}

The majority of people infected with severe acute respiratory syndrome coronavirus 2 (SARS-CoV-2) only develop mild symptoms, whereas a small subset of patients develop severe coronavirus disease 2019 (COVID-19) and some succumb to the disease ${ }^{1}$. Severe COVID-19 is a multisystemic disease, and thromboinflammation is emerging as a major cause of morbidity and mortality ${ }^{2}$. Clinical coagulopathy associated with severe COVID-19 is characterized by significant elevation of plasma D-dimer, moderate prolongation of prothrombin time and partial thromboplastin time, and mild thrombocytopenia ${ }^{3-7}$. Thromboinflammation in severe COVID-19 is characterized by increased plasma inflammatory markers, such as IL-6, tumor necrosis factor alpha, C-reactive protein, and activation of complement pathway ${ }^{3}$, and dysregulated activation of cellular components participating in inflammatory and coagulation responses that include platelets ${ }^{8}$, endothelium ${ }^{9}$, monocytes $^{10}$, and neutrophils ${ }^{11}$. Thrombotic manifestations range from arterial thrombosis, venous thromboembolism, to tissue micro thrombosis ${ }^{12}$ and occur in at least $20 \%$ of COVID-19 patients in intensive care unit (ICU), but perhaps more based on postmortem studies ${ }^{13}$. Of note, clinical manifestations, frequency, plasma biomarkers, and hematological changes seen in SARS-CoV-2-infected patients resemble those typical of heparin-induced thrombocytopenia and thrombosis $(\mathrm{HIT})^{14}$.

HIT is an antibody-mediated reaction in patients exposed to heparin, characterized by thrombocytopenia and venous and arterial thrombosis ${ }^{15}$. HIT is caused by formation of immunoglobulin Gs (IgGs), primarily $\lg G 1 s^{16}$, that recognize a complex formed between a platelet a-granule protein platelet factor 4 and heparin $(\mathrm{PF} 4 / \mathrm{H})$ or $\mathrm{PF} 4 /$ polyanion. In HIT, platelet-activating PF4/H-reactive antibodies drive a prothrombotic state through activation of Fc receptors on platelets ${ }^{17}$, monocytes ${ }^{18}$, and neutrophils ${ }^{19}$ and 
through activation of an unidentified molecule on endothelial cells ${ }^{20}$. "Spontaneous" HIT can arise in the absence of proximate heparin exposure and is similarly initiated by the formation of anti-PF4/H plateletactivating antibodies ${ }^{21}$.

We recently cloned from HIT patients a group of PF4/H-binding IgG1 antibodies that included plateletactivating clones ${ }^{22,23}$. PF4/H-reactive clones had longer heavy-chain complementarity determining region (HCDR3). Platelet-activating clones contained either an $\mathrm{RX}_{1-2} \mathrm{R} / \mathrm{K} / \mathrm{HX} \mathrm{X}_{1-2} \mathrm{R} / \mathrm{K} / \mathrm{H}$ or a string of 5 tyrosine residues in an unusually long HCDR3 ( $\geq 20$ amino acid residues), which we named RKH or $\mathrm{Y}_{5}$ motif respectively ${ }^{22,23}$. Interestingly, a group of antibodies reactive to the receptor-binding domain (RBD) of SARS-CoV-2 spike protein have longer HCDR3 ${ }^{24}$. Strikingly, clones possessing an RKH or $\mathrm{Y}_{5}$ motif have been identified among RBD-binding clones or as dominant clones in severe COVID-19 patients ${ }^{24,25}$. In the current study, we investigate whether severe COVID-19 patients develop platelet-activating and prothrombotic antibodies similar to those that drive the pathologic manifestations of HIT, however, independent of heparin exposure and whether RBD-specific antibodies can activate platelets with features similar to those cloned from HIT patients.

\section{Results}

\section{COVID-19 patients enrolled in the study}

Plasma and peripheral blood samples were collected from COVID-19 patients enrolled in "An Open label, Phase 2 Study Evaluating the Efficacy and Safety of High-Titer Anti-SARS-CoV2 plasma in hospitalized patients with COVID-19 infection" (NCT04354831). Patient information is presented in Table S1. Samples collected from 40 patients on day 0 of enrollment were analyzed. Plasma samples from 10 HIT patients (diagnosed based on clinical manifestation, positive PF4-reactive antibody assay, and positive serotonin release assay) and 8 deidentified healthy individuals were used as controls. The studies were approved by the Institutional Review Boards of the Medical College of Wisconsin and the Froedtert Hospital.

\section{Hospitalized COVID-19 patients developed PF4/H-reactive and platelet-activating antibodies that are independent of heparin exposure}

We investigated whether PF4/H-reactive antibodies are elicited in the setting of SARS-CoV-2 infection using the enzyme linked immunosorbent assay (ELISA) for PF4/H-reactive antibodies in hospitalized COVID-19 patients. Levels of plasma PF4/H-reactive IgG and IgM were both markedly higher in hospitalized COVID-19 patients than in healthy controls (Figs. 1A, 1B, and S1A), and PF4/H-reactive IgG level of the top $43 \%$ COVID-19 samples was similar to that in patients with HIT (Fig. 1A data in dashed rectangle). Excess heparin inhibited binding of plasma IgG and IgM antibodies in the COVID-19 patients, demonstrating the similarity of SARS-CoV-2 induced PF4/H-reactive antibodies to those elicited in the context of HIT (Figs. 1C and S1B). 
To examine whether platelet-activating antibodies are induced in the context of SARS-CoV-2 infection, we used the PF4-dependent P-selectin expression assay (PEA) ${ }^{26}$. In this assay, the ability of patient plasma to induce platelet activation is tested in the context of added PF4, and platelet activation is measured by the externalization of P-selectin. Plasma from COVID-19 inpatients induced higher levels of P-selectin expression on platelets compared to healthy controls (Fig. 2A). Since COVID-19 patient plasma has elevated levels of neutrophil extracellular traps (NETs) that are prominent in COVID-19 and are known to activate TLR927,28, we investigated whether TLR9 agonist CpG could co-stimulate platelets and thus enhance platelet responsiveness to platelet-activating antibodies in the PEA. Indeed, $\mathrm{CpG}$ substantially enhanced platelet responsiveness to platelet-activating antibodies cloned from HIT patients but had no effect on platelet activation when non-activating clones were added (Fig. S2). CpG co-stimulation in PEA $\left(P E A^{+C p G}\right)$ also markedly augmented platelet activation stimulated by plasma from COVID-19 patients, and this co-stimulatory effect of CpG was similarly observed in the context of HIT plasma (Fig. 2A). No platelet activation was induced by $\mathrm{CpG}$ in the context of plasma from healthy controls (Fig. 2A). We thus used $P E A^{+C p G}$ to measure platelet activation induced by plasma from 40 COVID-19 patients and found that markedly higher levels of P-selectin was induced by patient plasma than healthy controls (Fig. 2B and S3A). Platelet activation induced by the top $43 \%$ plasma samples mirrored those of plasma from HIT patients (Fig. 2B, data in dashed rectangle). PF4/H-reactive lgGs and P-selectin induction by all the COVID-19 plasma tested were positively correlated (Fig. S2B), and this correlation was intensified among the top $43 \%$ of COVID-19 plasma samples with stronger platelet-activating abilities (Fig. 2C), similar to HIT antibodies. IgG was purified from COVID-19 patient plasma with and without reactivity in the PEA ${ }^{+C p G}$

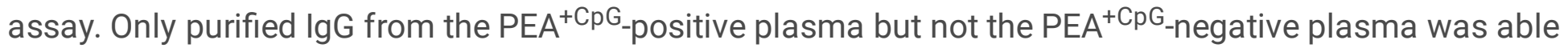
to activate platelets (Figs 2D and S3C). The activation was PF4-dependent and inhibited by IV.3 (a monoclonal FcgRIIA-blocking antibody), demonstrating the importance of PF4 and FcgRIIA in plateletactivation (Fig. 2D), similar to HIT antibodies. PF4 and FcgRIIA dependent platelet activation was also demonstrated in COVID-19 plasma (Fig. 2E). The similarity of SARS-CoV-2 induced platelet-activating antibodies to those developed in HIT was further demonstrated by the inhibition of antibody-induced platelet activation by excess heparin (Fig. 2E). Taken together, these data demonstrate that SARS-CoV-2 infection induces the production of platelet-activating antibodies with similar properties to antibodies produced in HIT.

Since a large number of hospitalized COVID-19 patients are exposed to unfractionated heparin (UFH) or low molecular weight heparin (LMWH) either prophylactically or therapeutically, development of PF4/Hreactive and platelet-activating antibodies in the COVID-19 patients in our study could be attributed to heparin exposure. We thus searched UFH/LMWH exposure history of these patients (Table S1). We found that $83 \%$ (14 patients) of patients tested positive for PF4/H-reactive or platelet-activating IgGs (17 patients) (Fig. 3) were exposed to UFH/LMWH only 1-2 days (30\%) or were heparin-naïve (53\%) before blood collection. Since PF4/H-reactive antibodies become marginally detectable at least 3 days after $\mathrm{UFH} / \mathrm{LMWH}$ exposure ${ }^{29,30}$, the development of PF4/H-reactive platelet-activating antibodies in hospitalized COVID-19 patients in the current study cannot be attributed to heparin. 


\section{Some RBD-binding antibodies can activate platelets}

Surprisingly, levels of PF4/H-reactive and RBD-reactive IgGs were positively correlated with each other (Figs. 4A and S4), suggesting potential cross-reactivity of a population of RBD-reactive IgGs with PF4/H. To investigate this possibility, we examined RBD and PF4/H cross-reactive B cells in the peripheral blood lymphocytes (PBMCs) of the COVID-19 patients by flow cytometry analysis. A high percentage of RBDbinding B cells also bound to PF4/H (39 $\pm 16 \%)$ while PF4/H-binding was low in those B cells that did not bind RBD $(2.2 \pm 1.8 \%)$ (Fig. 4B).

To investigate whether antibodies produced by the RBD-binding B cells activate platelets, we cloned antibodies from two patients, who had high-titer of RBD-specific antibodies and minimal heparin exposure. We sorted $\mathrm{RBD}^{+} \mathrm{IgG1}{ }^{+} \mathrm{B}$ cells (Fig. S5), and cloned 21 antibodies each from patient COVID39 (clones YZ1-17 and YZ39-42) and COVID40 (clones YZ18-38), respectively, using a single-cell PCR and expression cloning method. Among these, 4 platelet-activating clones were identified using PEA. Only a few of the antibodies cloned from the RBD-binding $B$ cells bound to RBD, whereas 18 clones bound to $\mathrm{PF} 4 / \mathrm{H}$ at a level comparable to those isolated from HIT patients ${ }^{22}$ (Figs. 4C and S6A). Of note, one (YZ5) of the 4 platelet-activating clones clearly bound to both PF4/H and RBD (Figs. 4C and 4D).

We further investigated whether higher affinity RBD-specific antibodies could activate platelets. Our recent studies in HIT have shown that PF4/H-binding and prothrombotic platelet-activating antibodies possess a k-chain with either an $\mathrm{RKH}$ or $\mathrm{Y}_{5}$ motif in their $\mathrm{HCDR}^{22,23}$. Interestingly, a large number of RBD-specific antibodies possess an RKH or $\mathrm{Y}_{5}$ motif ${ }^{24,25}$. Among antibodies cloned by Robbiani et al. from RBDbinding $B$ cells in convalescent COVID-19 patients ${ }^{25}, 15$ possess an RKH or $\mathrm{Y}_{5}$ motif with some clonal expansion (Table $\mathbf{S} 2$ and data not shown). To investigate the platelet-activating potential of these high affinity RBD-specific clones, we expressed, based on the published sequences, 6 RKH or $\mathrm{Y}_{5}$ motifpossessing clones that also used a k-chain ${ }^{25}$ (Table S2). Three of these antibodies were plateletactivating clones (Clones S1, S5, S6). Compared to the platelet-activating antibodies cloned in this study from the RBD ${ }^{+} \operatorname{lgG} 1^{+} \mathrm{B}$ cells ( $\mathrm{YZ}$ clones), clones S1, S5, and S6 bound much more strongly to RBD and induced equivalent platelet activation (Figs. 4C, 4E and S6B). Interestingly, S5 and S6 barely bound PF4/H (Fig. 4D). Thus, among the 7 platelet-activating antibodies identified from COVID-19 patients, 4 bound to RBD.

Of the 7 platelet-activating antibodies identified in the current study, activation by 6 antibodies was PF4 and FcgRIIA dependent and inhibited by high-dose heparin (Fig. 4F), indicating again that platelet activation by these antibodies function through a mechanism similar to that operates in HIT and also to that which has recently been described in vaccine-induced thrombocytopenia and thrombosis (VITT) following ChAdOx1n nCoV-19 and Ad26.COV2.S vaccination ${ }^{31-33}$. Different from other platelet-activating clones, clone S1 activated platelets in the absence of exogenously added PF4 and in the presence of high-dose heparin (Fig. 4F). In summary, a subset of RBD-specific antibodies induced by SARS-CoV-2 
activate platelets and have functional characteristics resembling those of the prothrombotic and pathogenic antibodies of HIT and VITT.

\section{RKH and $Y_{5}$ motifs are prominent in RBD-binding platelet-activating antibodies}

Of the three RBD-specific platelet-activating antibodies identified based on their possession of the RKH or $\mathrm{Y}_{5}$ motifs, two possessed an $\mathrm{Y}_{5}$ motif (Fig. 5A, Clones $\mathrm{S} 1$ and S5) and one possessed an RKH motif (Fig. $5 A$, Clone S6). Strikingly, in the four platelet-activating antibodies cloned from RBD-binding B cells in our current study, clones $Y Z 5$ and $Y Z 16$ contained the $Y_{5}$ and RKH sequences respectively and clone $Y Z 37$ contained the YXYYYY sequence that is very similar to the $Y_{5}$ motif. Different from the platelet-activating antibodies identified in HIT, which contained the RKH or $Y_{5}$ motif in an HCDR3 $\geq 20$ amino acid residues, these three platelet-activating antibodies cloned in COVID-19 had a much shorter HCDR3 ${ }^{22,23}$ (Fig. 5A). Thus, RKH or $\mathrm{Y}_{5}$ sequences appeared more important than the length of the HCDR3 in defining plateletactivating antibodies in COVID-19. In addition, whereas the 55 PF4/H-binding antibodies cloned from HIT patients exclusively used k-chains ${ }^{22,23}$, those cloned in COVID-19 patients, including one plateletactivating clone $Y Z 5$, also used I-chains (Fig. 5A). Hereafter, $R X_{1-2} R / K / H-X_{1-2} R / K / H$ and $Y Y Y Y Y$ sequences will be called RKH and $Y_{5}$ motifs, respectively, regardless of HCDR3 length. These data demonstrate that RBD-specific platelet-activating antibodies induced by SARS-CoV-2 infection bear structural motifs similar to those of platelet-activating antibodies induced in HIT.

Four of the 7 platelet-activating clones identified in the current study used $\mathrm{VH} 3$, including 2 which used VH3-30, and the remaining 3 used VH1, of which 1 used VH1-69 (Fig. 5A). VH3 and VH1 usage was also increased in the PF4/H-binding antibodies cloned from the RBD-binding B cells (Fig. S7). All of the platelet-activating clones and the majority of the PF4/H-binding clones displayed limited somatic mutation (Figs. 5B and S8). VH3 and VH1, in particular, VH3-30 and VH1-69 are over-represented in the spike-specific and RBD-specific antibodies induced in response to SARS-CoV-2 infection, and the majority of these antibodies have near germline sequences $24,25,34,35$. Thus, the structural signatures of the plateletactivating and PF4/H-binding clones that we have identified in COVID-19 patients are similarly enriched among the anti-spike and anti-RBD antibodies that have been previously reported.

\section{B cells possessing an RKH or $Y_{5}$ motif are expanded robustly in COVID-19 patients}

To investigate whether RKH or $\mathrm{Y}_{5}$ motif -possessing $\mathrm{B}$ cells are expanded in COVID-19 patients, we compared the frequency of $B$ cells possessing an RKH or $\mathrm{Y}_{5}$ motif in COVID-19 patients and healthy people based on the published data. We focused on IgG1 isotype given its relevance to HIT pathogenicity ${ }^{16}$. We analyzed the data generated by Galson et al. ${ }^{36}$ and Briney et al. ${ }^{37}$, which performed deep or ultra-deep sequencing of B-cell receptor repertoires of 19 COVID-19 patients and 10 healthy people. We found that $\lg \mathrm{G}^{+} \mathrm{B}$ cells were significantly increased in COVID-19 patients compared to healthy controls (Fig. 5C). VH sequences possessing an RKH or $\mathrm{Y}_{5}$ motif were present in IgG1 isotype in healthy controls (Fig. 5D, 3.3 $\pm 1.3 \%$ ), and were significantly increased in COVID-19 patients (Fig. 5D, 
$6.7 \pm 3.7 \%$ ). The abundance (in percentage) of the top ranked clones that possessed an RKH or $Y_{5}$ motif were significantly increased in IgG1 ${ }^{+} \mathrm{B}$ cells in COVID-19 patients relative to healthy controls (Figs. $\mathbf{5 E}$ and S9). Clonal expansion will reduce the diversity of B-cell receptor repertoire, which can be revealed by diversity indexes, including clonal diversity 50 (D50), Shannon index, and Simpson's index. We calculated D50, Shannon index, and Simpson's index of B cells possessing an RKH or $Y_{5}$ motif, and found that these indices of diversity were all significantly reduced in the $\mathrm{RKH}$ or $\mathrm{Y}_{5}$ possessing $\operatorname{lgG} 1^{+}$and $\lg \mathrm{G}^{+} \mathrm{B}$ cells in COVID-19 patients relative to healthy controls (Figs. 5F and S10). Thus, in COVID-19 patients, there is a robust expansion of $B$ cells possessing an $\mathrm{RKH}$ or $\mathrm{Y}_{5}$ motif.

\section{Discussion}

Here we demonstrate that in COVID-19, the induction of SARS-CoV-2 RBD-specific antibodies is closely associated with the induction of $\mathrm{PF} 4 / \mathrm{H}$ reactive platelet-activating antibodies. These $\mathrm{PF} / \mathrm{H}$-reactive antibodies bear striking similarities to the platelet-activating antibodies that drive the thrombotic manifestations of HIT and VITT. Specifically, COVID-19 patients had a markedly increased level of PF4/Hreactive platelet-activating IgGs, and the level of such antibodies correlated with RBD-reactive IgG level. A significant proportion of RBD-binding B cells in COVID-19 also recognized PF4/H. Among the 7 plateletactivating antibodies cloned from COVID-19 patients, 4 bound to the SARS-CoV-2 RBD, 6 had plateletactivating characteristics similar to prothrombotic antibodies of HIT and VITT, and 6 possessed structural

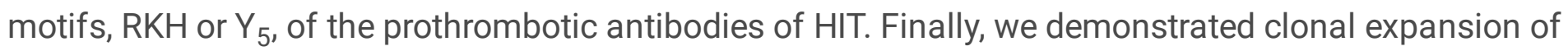
RKH or $Y_{5}$ motif -possessing B cells in the repertoire of COVID-19 patients.

Given the timing of heparin exposure, $\mathrm{PF} 4 / \mathrm{H}$-reactive platelet-activating antibodies in the plasma of the significant majority of hospitalized COVID-19 patients could not have been induced by heparin. In addition, among the 7 platelet-activating RBD-specific clones identified in the current study, 3 were from patient 39 who did not receive heparin and 3 were from out patients. Thus, the majority of plateletactivating antibodies detected were not elicited by heparin. Several other studies also detected increased PF4/H-reactive antibodies in COVID-19 patients in the context of heparin treatment and patients are suspected of developing "classical" HIT, i.e. heparin exposure-induced HIT ${ }^{38-42}$. A recent randomized trial reported that therapeutic dose of UFH and LMWH did not improve organ dysfunction in critically ill patients with COVID-1943. Thus, the induction of prothrombotic platelet-activating antibodies by heparin and SARS-CoV-2 individually and cooperatively and their association with critical illness merits careful investigation.

Althaus et al. reported that IgG fractions from COVID-19 patients induced an FcgRIIA-dependent plateletactivation, specifically procoagulant apoptotic platelet formation, and such activity in the plasma appeared to contribute to disease severity ${ }^{44,45}$. However, it is not clear whether these antibodies are heparin-dependent or not. We found that the levels of platelet-activating antibodies in COVID-19 plasma relative to the healthy controls were significantly elevated but still lower than those in HIT patients. Costimulation of platelets with the TLR9 agonist CpG and plasma from hospitalized COVID-19 patients 
induced platelet activation to a level similar to that induced by HIT plasma. Such elevated but subthreshold level of platelet-activating antibodies in severe COVID-19 could be sufficient to activate platelets and induce a prothrombotic state in the presence of strong inflammatory stimuli, such as TLR9 agonist NETs, in severe COVID-19 patients.

In addition to inflammatory signals, circulating plasma PF4 and PF4 released from activated platelets in the context of tissue damage during SARS-CoV-2 infection may also potentiate the prothrombotic functions of platelet-activating antibodies. In this context, platelet-activation by PF4/H-reactive antibodies in HIT appears to be largely dependent on neo-epitope formation when PF4 associates with cell surface glycosaminoglycan ${ }^{46,47}$. Some studies have shown an increase of circulating plasma PF4 in patients with severe-to-critical COVID-1912, but others have demonstrated a reduction of PF4 in patients with poor prognosis ${ }^{48}$. Activated platelets and platelet-secretory cargo, including PF4, are detected in the airways of patients with severe COVID-1949. The level of PF4 is also increased in bronchoalveolar lavage, peripheral blood, and serum of SARS-CoV-2-infected rhesus macaques ${ }^{50}$. Plasma PF4 is strongly associated platelet activation, and the high degree of platelet activation and thrombocytopenia associated with severe COVID-19, will affect plasma PF4 levels during disease progression. A better understanding of local and circulating concentrations of plasma factors, including TLR9 and PF4, that potentiate the platelet response should help delineate the prothrombotic role of such antibodies in COVID-19 patients.

Although platelet-activating antibodies cloned from COVID-19 patients bear structural and plateletactivating characteristics resembling the antibodies in HIT, there are some differences between the two groups of antibodies. 3 platelet-activating antibodies from COVID-19 patients barely bound PF4/H, which indicates that platelet-activation is not necessarily dependent on antibody recognition of $\mathrm{PF} / \mathrm{H}$. The four platelet-activating antibodies cloned from RBD-binding B cells in COVID-19 patients (YZ clones) had a much shorter HCDR3 than those isolated from HIT patients, which have an HCDR3 $\geq 20$ amino acid residues $^{22,23}$. Thus, $\mathrm{RKH}$ or $\mathrm{Y}_{5}$ sequences appear more important than the length of the HCDR3 in defining platelet-activating antibodies in COVID-19. Whereas all 55 PF4/H-binding antibodies cloned from HIT patients exclusively used k-chains ${ }^{22,23}$, those cloned in COVID-19 patients, including one plateletactivating clone YZ5, also used I-chains. These structural differences of platelet-activating antibodies may be attributable to different B-cell stimulating antigen(s) in HIT and COVID-19 patients, or alternatively, may be due to limited number of clones identified in both diseases.

A large proportion of RBD-binding B cells cross-reacted with PF4/H (Fig. 1B). However, whereas about $40 \%$ of antibodies cloned from RBD binding $B$ cells could interact with PF4/H, the majority bound RBD only very weakly. We observed that PF4/H-reactive antibodies are largely auto-reactive and poly-reactive 23 (Zhu et. Al., unpublished study) and propose that innate B cells produce these auto-reactive plateletactivating antibodies and are controlled by intrinsic and extrinsic tolerance mechanisms as demonstrated in mice ${ }^{51-53}$. We postulate that even with very low affinity to RBD, these RBD and PF4/H cross-reactive B cells can be activated by RBD in the presence of high inflammatory signals provided by SARS-CoV-2 infection ${ }^{54}$. Whereas the antibodies cloned in our current study (YZ clones), including several platelet- 
activating clones, bound to RBD only very weakly, the three platelet-activating antibodies identified based on possession of an RKH or $\mathrm{Y}_{5}$ motif (S clones) bound to RBD with intermediate to high affinity. Thus, in COVID-19, a subset of B cells with variable affinity to the RBD of the SARS-CoV-2 spike protein are activated and generate antibodies capable of PF4-dependent platelet activation. Some of these PF4/H and RBD cross-reactive antibodies have functional and structural characteristic analogous to antibodies observed in HIT and would be similarly expected to drive local and systemic thrombosis and contribute to the thrombotic complications prominent in SARS-CoV-2 infection. Given the recent description of a HITlike clinical syndrome VITT following ChAdOx 1 nCov-19 and Ad26.COV2.S vaccination ${ }^{31-33}$, our findings also provide a potential mechanistic explanation for the clinical observations. We posit that studies of the structural and functional characteristics of the antibodies and B cells generated in VITT may demonstrate similarities of the pathogenic antibodies of VITT with those that we identify here in the setting of COVID19.

In summary, SARS-CoV-2 infection activates RBD-specific B cells to make HIT-like platelet-activating antibodies that are defined by the RKH and $Y_{5}$ motif. These antibodies are capable of PF4-dependent platelet activation and may therefore contribute to the thrombotic complications seen in SARS-CoV-2 infection as they do in HIT.

\section{Declarations}

Acknowledgment We thank Dr. Patrick Wilson (University of Chicago) for helping us establishing the single B-cell PCR and expression cloning system, Dr. Peter Newman (Versiti) for providing the purified anti-FcgRIIA antibody IV.3, and Trudy Holyst and Benedetta Bonacci for excellent technical support. The work was supported by NIH HL148120 (R.W.), CTSI UL1TR001436 (R.W.), ACTIV4 (R.W.), NIH AI079087 (D.W.), HL130724 (D.W.), and AHA 20PRE35210461 (W.Z.). Advancing a Healthier Wisconsin Endowmwnt (GCW).

Author contributions R.W. designed the study. W.Z. performed all the plasma antibody and majority of the monoclonal antibody ( $\mathrm{mAb}$ ) analysis and cloned some mAbs; Y. Zheng cloned majority of the mAbs, performed some of the functional and all the sequence analysis of the mAbs, and performed the B-cell phenotype analysis. M.Y. performed the B-cell receptor repertoire analysis. J.W. cloned and made the recombinant human PF4 protein. Y. Zhang helped with the mAb expression. W.Z., Y. Zheng, M.Y., C.N., P.T., and R.J. processed the patient samples and established the sample bank for the COVID-19 study. M.B.G. was in charge of and P.H. helped with the convalescent plasma trial of COVID-19 patients. M.B.G. provided the patient information. M.B.G. R.A, S.J., G.C.W., W.C, L.B.K, R.A. and D.W. had valuable input and edited the manuscript. R.W. wrote the manuscript with the help of W.Z., Y. Zheng, and M.Y.

Authors declare no conflict of interest.

\section{References}


Wu, Z. \& McGoogan, J. M. Characteristics of and Important Lessons From the Coronavirus Disease 2019 (COVID-19) Outbreak in China: Summary of a Report of 72314 Cases From the Chinese Center for Disease Control and Prevention. JAMA 323, 1239-1242, doi:10.1001/jama.2020.2648 (2020).

2 Gu, S. X. et al. Thrombocytopathy and endotheliopathy: crucial contributors to COVID-19 thromboinflammation. Nature reviews. Cardiology, doi:10.1038/s41569-020-00469-1 (2020).

3 Zhou, F. et al. Clinical course and risk factors for mortality of adult inpatients with COVID-19 in Wuhan, China: a retrospective cohort study. Lancet, doi:10.1016/S0140-6736(20)30566-3 (2020).

4 Liao, D. et al. Haematological characteristics and risk factors in the classification and prognosis evaluation of COVID-19: a retrospective cohort study. Lancet Haematol, doi:10.1016/S23523026(20)30217-9 (2020).

5 Shah, S. et al. Elevated D-Dimer Levels are Associated with Increased Risk of Mortality in COVID19: A Systematic Review and Meta-Analysis. Cardiol. Rev., doi:10.1097/CRD.0000000000000330 (2020).

6 Tang, N., Li, D., Wang, X. \& Sun, Z. Abnormal coagulation parameters are associated with poor prognosis in patients with novel coronavirus pneumonia. Journal of thrombosis and haemostasis : JTH 18, 844-847, doi:10.1111/jth.14768 (2020).

7 Guan, W. J. et al. Clinical Characteristics of Coronavirus Disease 2019 in China. N. Engl. J. Med. 382, 1708-1720, doi:10.1056/NEJMoa2002032 (2020).

8 Manne, B. K. et al. Platelet Gene Expression and Function in COVID-19 Patients. Blood, doi:10.1182/blood.2020007214 (2020).

9 Goshua, G. et al. Endotheliopathy in COVID-19-associated coagulopathy: evidence from a singlecentre, cross-sectional study. Lancet Haemato/ 7, e575-e582, doi:10.1016/S2352-3026(20)30216-7 (2020).

10 Merad, M. \& Martin, J. C. Pathological inflammation in patients with COVID-19: a key role for monocytes and macrophages. Nature reviews. Immunology 20, 355-362, doi:10.1038/s41577-020-0331-4 (2020).

11 Middleton, E. A. et al. Neutrophil Extracellular Traps (NETs) Contribute to Immunothrombosis in COVID-19 Acute Respiratory Distress Syndrome. Blood, doi:10.1182/blood.2020007008 (2020).

12 Ackermann, M. et al. Pulmonary Vascular Endothelialitis, Thrombosis, and Angiogenesis in Covid19. N. Engl. J. Med. 383, 120-128, doi:10.1056/NEJMoa2015432 (2020).

13 Wichmann, D. et al. Autopsy Findings and Venous Thromboembolism in Patients With COVID-19: A Prospective Cohort Study. Ann. Intern. Med. 173, 268-277, doi:10.7326/M20-2003 (2020). 
14 Warkentin, T. E. \& Kaatz, S. COVID-19 versus HIT hypercoagulability. Thromb. Res. 196, 38-51, doi:10.1016/j.thromres.2020.08.017 (2020).

15 Arepally, G. M. Heparin-induced thrombocytopenia. Blood 129, 2864-2872, doi:10.1182/blood2016-11-709873 (2017).

16 Suh, J. S., Malik, M. I., Aster, R. H. \& Visentin, G. P. Characterization of the humoral immune response in heparin-induced thrombocytopenia. Am. J. Hematol. 54, 196-201, doi:10.1002/(SICI)10968652(199703)54:3<196::AID-AJH4>3.0.C0;2-R [pii] (1997).

17 Kelton, J. G. et al. Heparin-induced thrombocytopenia: laboratory studies. Blood 72, 925-930 (1988).

18 Rauova, L. et al. Monocyte-bound PF4 in the pathogenesis of heparin-induced thrombocytopenia. Blood 116, 5021-5031, doi:10.1182/blood-2010-03-276964 (2010).

19 Perdomo, J. et al. Neutrophil activation and NETosis are the major drivers of thrombosis in heparin-induced thrombocytopenia. Nature communications 10, 1322, doi:10.1038/s41467-019-09160-7 (2019).

20 Cines, D. B., Tomaski, A. \& Tannenbaum, S. Immune endothelial-cell injury in heparin-associated thrombocytopenia. N. Engl. J. Med. 316, 581-589, doi:10.1056/NEJM198703053161004 (1987).

21 Warkentin, T. E., Basciano, P. A., Knopman, J. \& Bernstein, R. A. Spontaneous heparin-induced thrombocytopenia syndrome: 2 new cases and a proposal for defining this disorder. Blood 123, 36513654, doi:10.1182/blood-2014-01-549741 (2014).

22 Zhu, W. et al. Antibody Cloning Identifies Pathogenic and Non-Pathogenic Antibodies in HeparinInduced Thrombocytopenia and Defines the Molecular Signatures That Differentiate the Two Types of Antibodies. Blood 134, 439-439, doi:10.1182/blood-2019-126097 (2019).

23 Zhu, W. et al. Polyreactivity and Somatic Hypermutation Analysis Reveals the Innate B Cell Origin of Human PF4/Heparin Reactive Antibodies. Blood 136, 34-35, doi:10.1182/blood-2020-138781 (2020).

24 Brouwer, P. J. M. et al. Potent neutralizing antibodies from COVID-19 patients define multiple targets of vulnerability. Science $369,643-650$, doi:10.1126/science.abc5902 (2020).

25 Robbiani, D. F. et al. Convergent antibody responses to SARS-CoV-2 in convalescent individuals. Nature 584, 437-442, doi:10.1038/s41586-020-2456-9 (2020).

26 Samuelson Bannow, B. T. et al. A prospective, blinded study of a PF4-dependent assay for HIT diagnosis. Blood, doi:10.1182/blood.2020008195 (2020). 
27 Zuo, Y. et al. Neutrophil extracellular traps in COVID-19. JCI Insight 5, doi:10.1172/jci.insight.138999 (2020).

28 Roers, A., Hiller, B. \& Hornung, V. Recognition of Endogenous Nucleic Acids by the Innate Immune System. Immunity 44, 739-754, doi:10.1016/j.immuni.2016.04.002 (2016).

29 Warkentin, T. E., Sheppard, J. A., Moore, J. C., Cook, R. J. \& Kelton, J. G. Studies of the immune response in heparin-induced thrombocytopenia. Blood 113, 4963-4969, doi:10.1182/blood-2008-10186064 (2009).

30 Greinacher, A., Kohlmann, T., Strobel, U., Sheppard, J. A. \& Warkentin, T. E. The temporal profile of the anti-PF4/heparin immune response. Blood 113, 4970-4976, doi:blood-2008-08-173062 [pii]

10.1182/blood-2008-08-173062 [doi] (2009).

31 Greinacher, A. et al. Thrombotic Thrombocytopenia after ChAdOx1 nCov-19 Vaccination. N. Engl. J. Med., doi:10.1056/NEJMoa2104840 (2021).

32 Schultz, N. H. et al. Thrombosis and Thrombocytopenia after ChAdOx1 nCoV-19 Vaccination. N. Engl. J. Med., doi:10.1056/NEJMoa2104882 (2021).

33 Muir, K. L., Kallam, A., Koepsell, S. A. \& Gundabolu, K. Thrombotic Thrombocytopenia after Ad26.COV2.S Vaccination. N. Engl. J. Med., doi:10.1056/NEJMc2105869 (2021).

34 Kreer, C. et al. Longitudinal Isolation of Potent Near-Germline SARS-CoV-2-Neutralizing Antibodies from COVID-19 Patients. Cell 182, 1663-1673, doi:10.1016/j.cell.2020.08.046 (2020).

35 Seydoux, E. et al. Analysis of a SARS-CoV-2-Infected Individual Reveals Development of Potent Neutralizing Antibodies with Limited Somatic Mutation. Immunity 53, 98-105 e105, doi:10.1016/j.immuni.2020.06.001 (2020).

36 Galson, J. D. et al. Deep Sequencing of B Cell Receptor Repertoires From COVID-19 Patients Reveals Strong Convergent Immune Signatures. Frontiers in immunology 11, 605170, doi:10.3389/fimmu.2020.605170 (2020).

37 Briney, B., Inderbitzin, A., Joyce, C. \& Burton, D. R. Commonality despite exceptional diversity in the baseline human antibody repertoire. Nature 566, 393-397, doi:10.1038/s41586-019-0879-y (2019).

38 Nazy, l. et al. Platelet-activating immune complexes identified in critically ill COVID-19 patients suspected of heparin-induced thrombocytopenia. Journal of thrombosis and haemostasis : JTH, doi:10.1111/jth.15283 (2021).

39 Brodard, J. et al. COVID-19 patients often show high-titer non-platelet-activating anti-PF4/heparin IgG antibodies. Journal of thrombosis and haemostasis : JTH, doi:10.1111/jth.15262 (2021). 
40 May, J. E., Siniard, R. C. \& Marques, M. The challenges of diagnosing heparin-induced thrombocytopenia in patients with COVID-19. Res Pract Thromb Haemost, doi:10.1002/rth2.12416 (2020).

41 Patell, R. et al. Heparin induced thrombocytopenia antibodies in COVID-19. Am. J. Hematol., doi:10.1002/ajh.25935 (2020).

42 Daviet, F. et al. Heparin-Induced Thrombocytopenia in Severe COVID-19. Circulation 142, 18751877, doi:10.1161/CIRCULATIONAHA.120.049015 (2020).

43 Al-Samkari, H. et al. Thrombosis, Bleeding, and the Observational Effect of Early Therapeutic Anticoagulation on Survival in Critically III Patients With COVID-19. Ann. Intern. Med., doi:10.7326/M206739 (2021).

44 Althaus, K. et al. Antibody-induced procoagulant platelets in severe COVID-19 infection. Blood, doi:10.1182/blood.2020008762 (2020).

45 Jobe, S. M. \& Wen, R. Another front in COVID-19's perfect storm. Blood 137, 1006-1007, doi:10.1182/blood.2020010459 (2021).

46 Rauova, L. et al. Role of platelet surface PF4 antigenic complexes in heparin-induced thrombocytopenia pathogenesis: diagnostic and therapeutic implications. Blood 107, 2346-2353, doi:10.1182/blood-2005-08-3122 (2006).

47 Padmanabhan, A. et al. Heparin-independent, PF4-dependent binding of HIT antibodies to platelets: implications for HIT pathogenesis. Blood 125, 155-161, doi:10.1182/blood-2014-06-580894 (2015).

48 Shen, B. et al. Proteomic and Metabolomic Characterization of COVID-19 Patient Sera. Cell 182, 59-72 e15, doi:10.1016/j.cell.2020.05.032 (2020).

49 Hottz, E. D. et al. Platelet activation and platelet-monocyte aggregate formation trigger tissue factor expression in patients with severe COVID-19. Blood 136, 1330-1341, doi:10.1182/blood.2020007252 (2020).

50 Aid, M. et al. Vascular Disease and Thrombosis in SARS-CoV-2-Infected Rhesus Macaques. Cell 183, 1354-1366 e1313, doi:10.1016/j.cell.2020.10.005 (2020).

51 Zheng, Y. et al. B-cell tolerance regulates production of antibodies causing heparin-induced thrombocytopenia. Blood 123, 931-934, doi:10.1182/blood-2013-11-540781 (2014).

52 Zheng, Y. et al. Regulatory T Cells Control PF4/Heparin Antibody Production in Mice. J Immunol, doi:10.4049/jimmunol.1900196 (2019). 
53 Zheng, Y. et al. Critical role for mouse marginal zone B cells in PF4/heparin antibody production. Blood 121, 3484-3492, doi:10.1182/blood-2013-01-477091 (2013).

54 Tay, M. Z., Poh, C. M., Renia, L., MacAry, P. A. \& Ng, L. F. P. The trinity of COVID-19: immunity, inflammation and intervention. Nature reviews. Immunology 20, 363-374, doi:10.1038/s41577-020-03118 (2020).

\section{Figures}

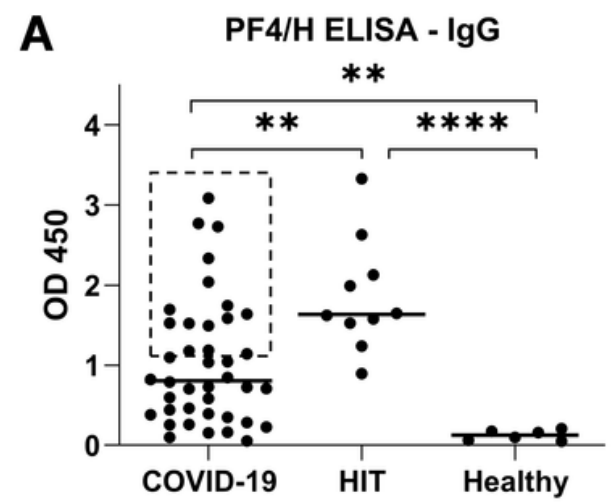

B

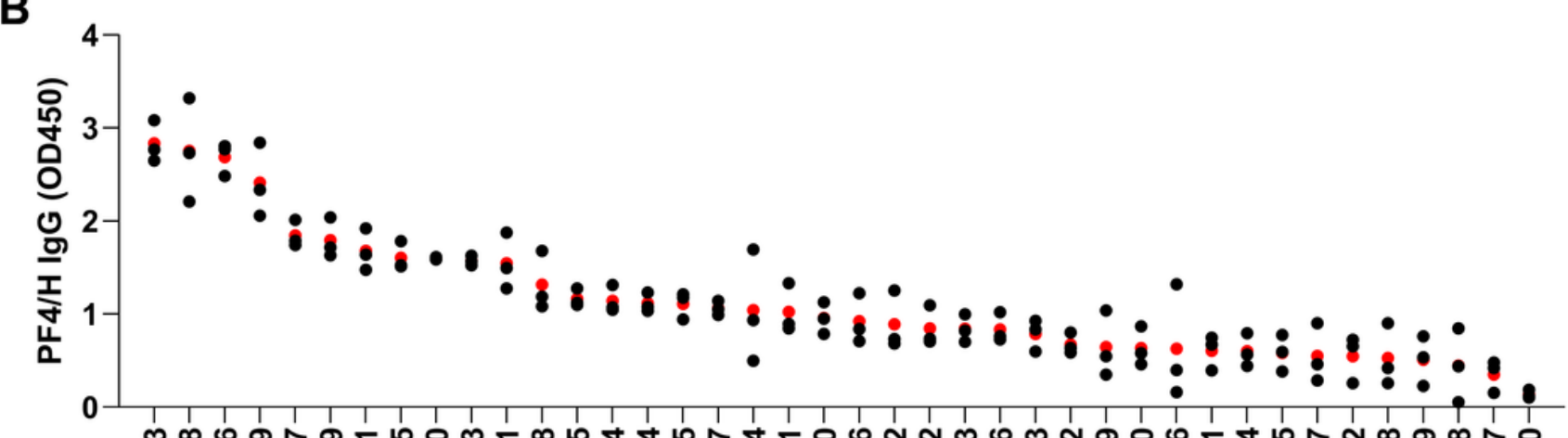

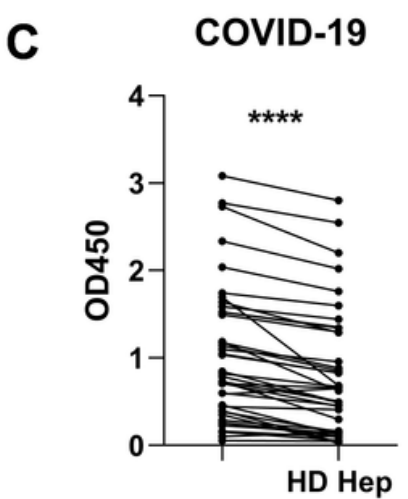

PF4/H IgG

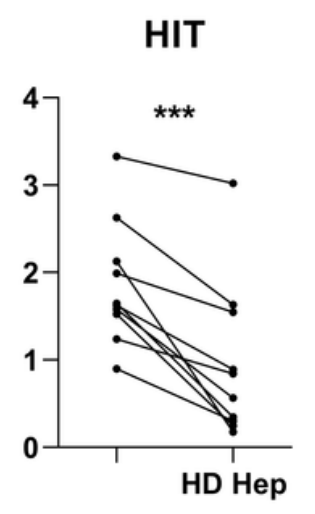

PF4/H IgG
Healthy

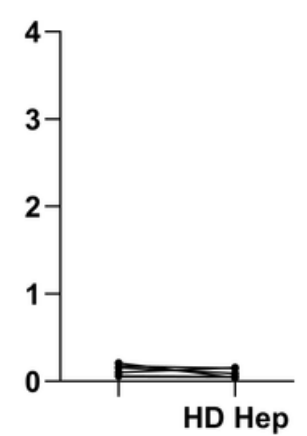

PF4/H IgG

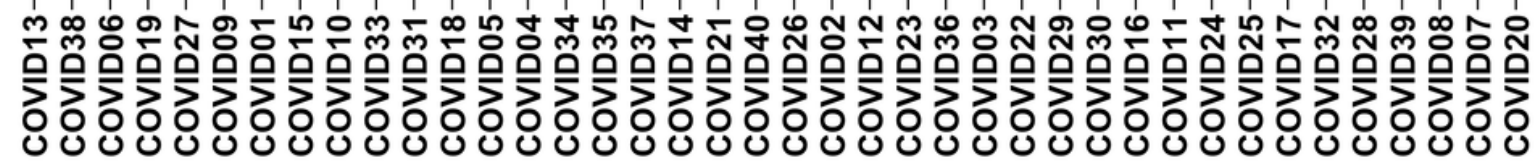

Fig. 1

Figure 1

Hospitalized COVID-19 patients develop PF4/H-reactive IgG antibodies. A. PF4/H-reactive IgG antibodies were increased in COVID-19 patients compared to healthy controls. B. A plot of 3 independent measurements of PF4/H-reactive IgG level in COVID-19 patient plasma. The average level of PF4/Hreactive IgG for each patient was presented by the red dots. C. Binding of IgG antibodies to PF4/H was inhibited by high-dose (HD) heparin. Plasma samples from 40 hospitalized COVID-19 patients were tested. $100 \mathrm{x}$ diluted plasma was used in the assays. Data shown in $A$ and $C$ were representative of 3 independent experiments. P-values were calculated by unpaired $(A)$ and paired $(C)$ two-tailed Student's ttest. *: $p<0.05, * *: p<0.01, * \star *: p<0.001, * \star \star *: p<0.0001$. The same symbols representing the corresponding $p$-values were used throughout the paper unless stated otherwise. 
A B C
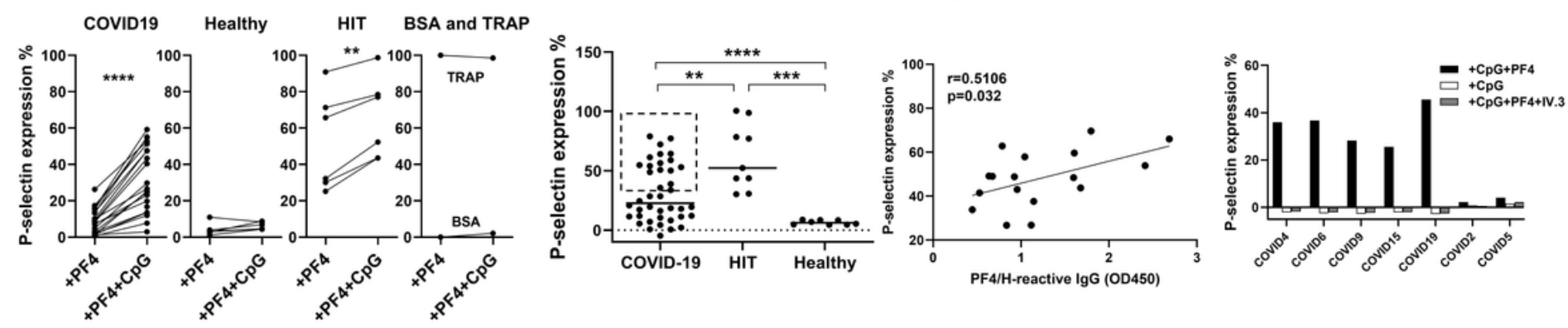

E

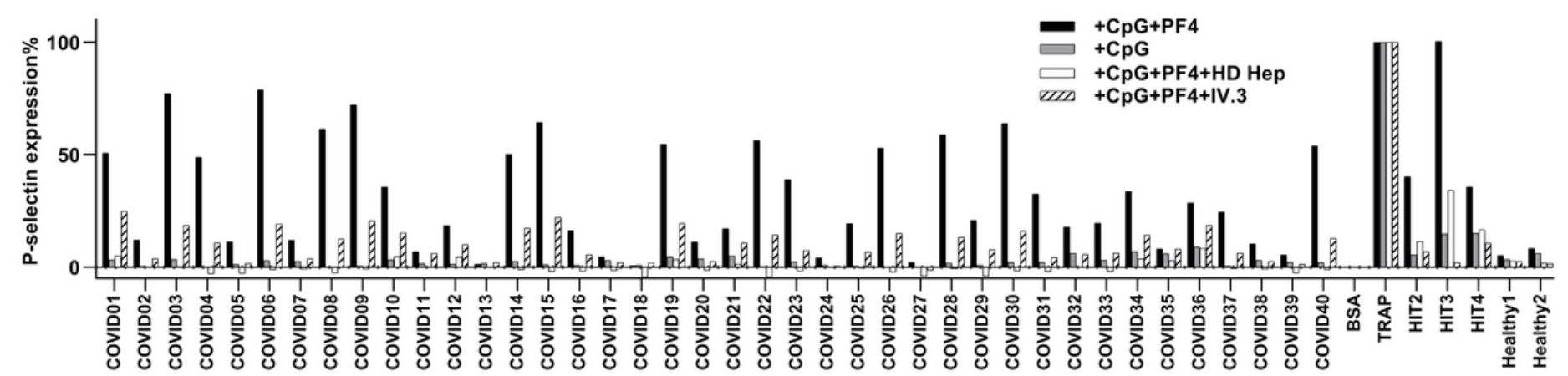

Fig. 2

Figure 2

Hospitalized COVID-19 patients have HIT-like platelet-activating antibodies. A. CpG treatment significantly enhanced platelet activation by COVID-19 and HIT plasma, but not healthy plasma in PEA. TRAP (thrombin receptor activating peptide) was used as a positive control. B. Plasma from COVID-19 patients could activate platelets in the PEA+CpG. C. The top 40\% PEA+CpG-positive samples from COVID-19 patients were correlated with the level of PF4/H-reactive IgGs. D. Purified IgG antibodies from PEA+CpGpositive but not PEA+CpG-negative COVID-19 plasma samples were able to activate platelets in the PEA+CpG. E. Platelet activation by COVID-19 plasma was PF4 and FcyRIIA-dependent and inhibited by HD Heparin. Plasma samples from 40 hospitalized COVID-19 patients (COVID1-40) were tested by the PEA+CpG in the presence or absence of exogenously added PF4, or in the presence of IV.3 or HD heparin. Plasma samples from HIT patients (HIT2-4) and healthy people (Healthy 1 and 2) were included as controls. Figs. A-C and E represent 3 independent experiments and Fig. D is a representative of 2 independent experiment.
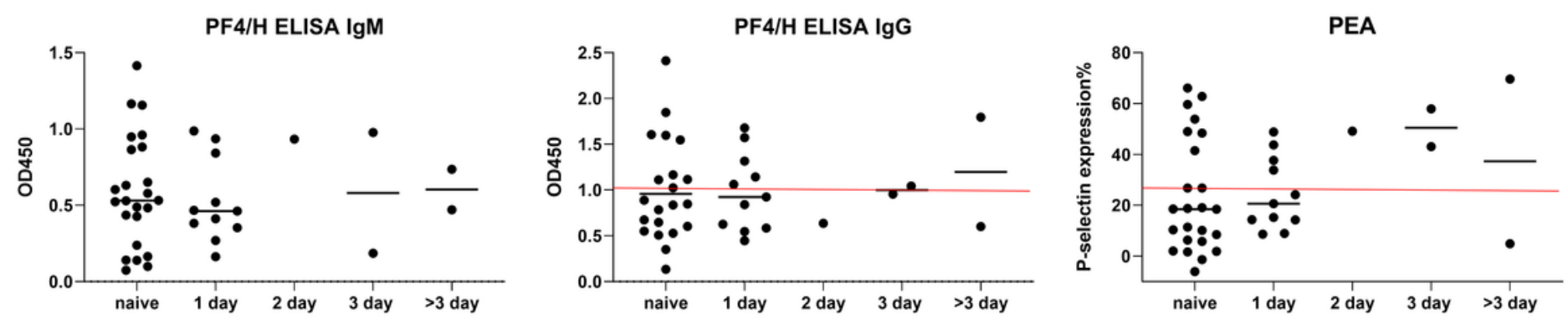

Days after UFH/LMWH exposure

Figure 3 
Hospitalized COVID-19 patients develop PF4/H-reactive and platelet-activating IgGs independent of UFH/LMWH exposure. Heparin-naïve refers to that patients were not exposed to UFH/LMWH at the time of blood collection for antibody measurement. The red lines represent the positive cutoff lines of the level of PF4/H-reactive IgGs or platelet-activating IgGs.
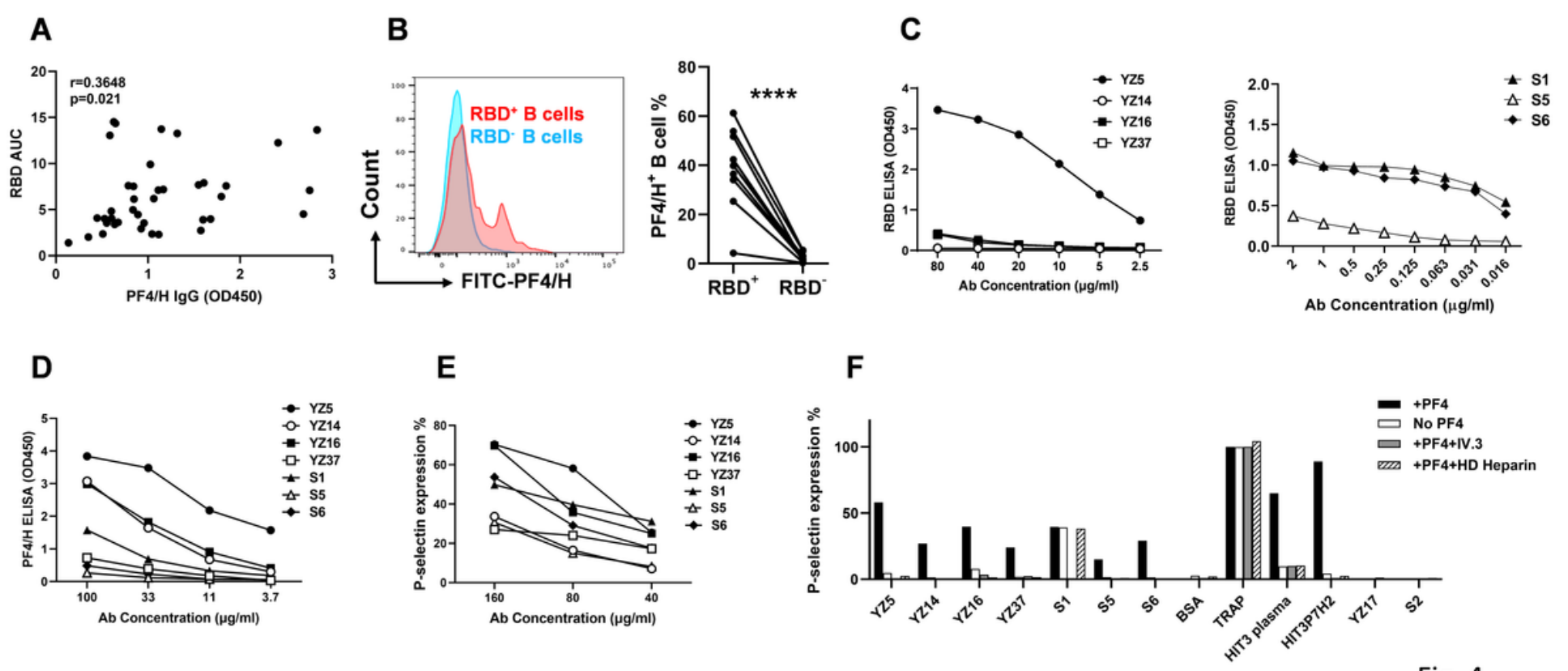

Fig. 4

\section{Figure 4}

Some RBD-specific antibodies are platelet-activating antibodies. A. Levels of PF4/H-reactive and RBDreactive IgGs in the plasma of COVID-19 patients were significantly correlated with each other. B. A large proportion of RBD-binding B cells also recognized PF4/H. C. RBD-binding curves of the 7 plateletactivating antibodies. D. PF4/H-binding curves of the 7 platelet-activating antibodies. E. PEA curves of the 7 platelet-activating antibodies. F. The activation properties of the seven platelet-activating antibodies. PEA was performed under the regular PEA condition, in the absence of exogenously added PF4, or in the presence of IV. 3 or HD heparin. HIT3P7H2 is a platelet-activating antibody cloned from an HIT patient (HIT3). Data is a representative of 2-4 independent experiments. 
A

\begin{tabular}{llcc}
\hline Clone & \multicolumn{1}{c}{ HCDR3 } & VH gene & L-chain \\
\hline YZ5 & ARTPGRISRYYYYYGMDV & VH3-33 & $\lambda$ \\
YZ14 & ARATRVCGGSCYEY & VH1-2 & $\kappa$ \\
YZ16 & AKRQRWLHNQAPFDY & VH3-30 & $\kappa$ \\
YZ37 & AKGGRYSYYYYGMDV & VH3-30 & $\kappa$ \\
& & & \\
S1 & ARGNRLLYCSSTSCYLDAVRQGYYYYYYMDV & VH1-69 & $\kappa$ \\
S5 & VETNLWFGEDNYYYYYGMDV & VH3-23 & $\kappa$ \\
S6 & ARVGHARGVITGGDYFYYGMDV & VH1-18 & $\kappa$ \\
\hline
\end{tabular}

D

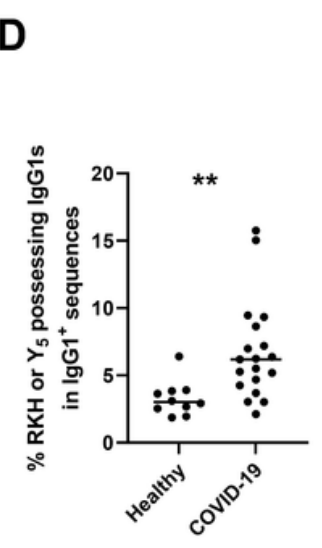

E

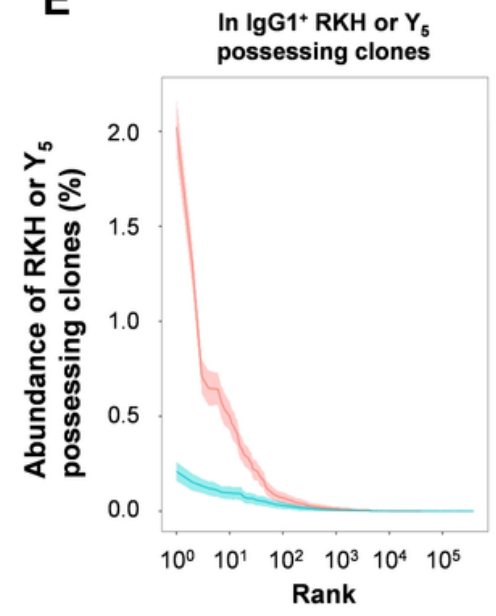

B

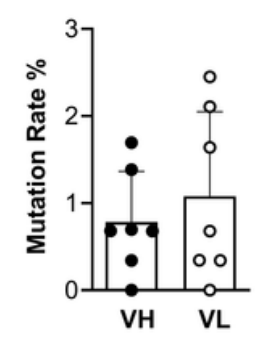

C

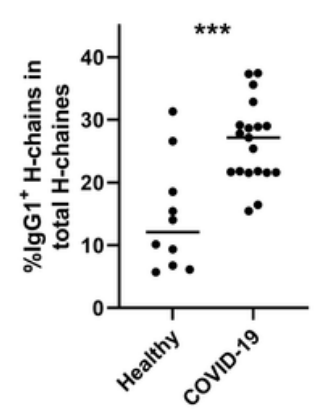

$\mathbf{F}$

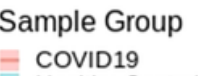

= COVID19
Healthy Control

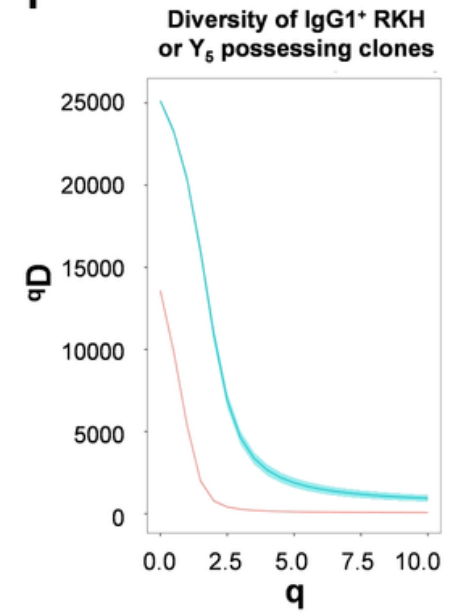

Sample Group

= COVID19

= Healthy Control

Fig. 5

\section{Figure 5}

RKH and $Y 5$ motifs are prominent in platelet-activating antibodies cloned from RBD-binding $B$ cells in COVID-19 patients. A. The HCDR3 sequence of the platelet-activating antibodies cloned from RBD-binding B cells in COVID-19 patients. The RKH and Y5 motifs were shown in bold red or blue font respectively. B. The 7 platelet-activating antibodies have low mutation rate. C. IgG1+B cells are significantly increase in COVID-19 patients compared to healthy controls. D. IgG1+ B cells that possess an RKH or Y5 motif are significantly more prevalent in COVID-19 patients compared to healthy controls. E. The abundance of the top ranked IgG1 + B cells that possess an RKH or Y5 motif are significantly higher in COVID-19 patients relative to healthy controls. The rank abundance distributions were calculated within the total reads of IgG1+ sequences that possessed an RKH or Y5 motif in the 19 COVID-19 patients and 10 healthy people with equivalent sampling size. F. Clonal diversity of the $\lg \mathrm{G} 1+\mathrm{B}$ cells that possess an RKH or Y5 motif are significantly reduced in COVID-19 patients relative to healthy controls. Clonal Diversity analysis was performed using the generalized diversity index proposed by Hill with uniform resampling from the total sequences possessing an RKH or Y 5 motif from 19 COVID-19 patients or 10 healthy people to correct for sequencing depth.

\section{Supplementary Files}

This is a list of supplementary files associated with this preprint. Click to download. 
- suplementarydata20210422.pdf

- supplementrayTables.docx 